RUNNING HEAD: SOCIO-AFFECTIVE DEFICITS IN ASD

\title{
White Matter Structure in the Uncinate Fasciculus: Implications for Socio-Affective Deficits in Autism Spectrum Disorder
}

Samson, Andrea C.* ${ }^{\mathrm{a}, \mathrm{b}}$, Dougherty, Robert F. ${ }^{\mathrm{c}}$, Lee, Ihno A. ${ }^{\mathrm{b}}$, Phillips, Jennifer M. ${ }^{\mathrm{d}}$, Gross, James J. ${ }^{\mathrm{b}}$, and Hardan, Antonio Y. ${ }^{\mathrm{d}}$

${ }^{a}$ Swiss Center for Affective Sciences (CISA), Campus Biotech, University of Geneva, Chemin des Mines 9, 1202 Geneva, Switzerland

${ }^{\mathrm{b}}$ Department of Psychology, Stanford University, 450 Serra Mall, Stanford, CA, 94305 USA

${ }^{\mathrm{c}}$ Stanford Center for Cognitive and Neurobiological Imaging, Stanford University, 450 Serra Mall, Stanford, CA, 94305 USA

${ }^{\mathrm{d}}$ Department of Psychiatry and Behavioral Sciences, Stanford University School of Medicine, 401 Quarry Road, Stanford, CA, USA

Corresponding author: Andrea Samson, Swiss Center for Affective Sciences (CISA), Campus Biotech, University of Geneva, Chemin des Mines 9, 1202 Geneva, Switzerland; andrea.samson@unige.ch; phone: +41223790932

Words (text): 4548; Words (abstract): 189; 2 Tables; 3 Figures 


\begin{abstract}
Individuals with Autism Spectrum Disorder (ASD) have social and communication deficits and difficulties regulating emotions. The brain bases of these socio-affective deficits are not yet clear, but one candidate is structural connectivity in the left uncinate fasciculus, which connects limbic temporal and frontal areas thought to be involved in socio-affective processing. In this study, we assessed white matter structure in the left and right uncinate fasciculus in 18 highfunctioning individuals with ASD and 18 group-matched typically developing (TD) controls using Diffusion Tensor Imaging. To test specificity of the associations, we also examined the association between both uncinate fasciculi and restricted and repetitive behaviors. Compared to TD individuals, individuals with ASD had significantly lower fractional anisotropy (FA) in the left and right uncinate. Group status significantly moderated the association between left uncinate and socio-affective deficits, indicating that within the ASD group, FA was associated with socio-affective deficits: Individuals with ASD with lower FA in the left uncinate had significantly more social and emotion regulation deficits. There was no association with restricted and repetitive behaviors. This study provides evidence that the left uncinate may play a critical role in socio-affective skills in individuals with ASD.
\end{abstract}

Keywords: Autism Spectrum Disorder; social deficits; emotion regulation; uncinate fasciculus; Diffusion Tensor Imaging 


\section{Introduction}

Individuals with Autism Spectrum Disorder (ASD) have social and communication deficits as well as increased restricted and repetitive behaviors (APA, 2013). It is now clear that ASD is also often associated with pronounced difficulties regulating emotions (Laurent and Rubin, 2004; Mazefsky et al., 2013, 2014; Samson et al., 2012, 2014, 2015a). At a neurobiological level, ASD has been associated with abnormal white matter tissue structure in several pathways in the brain, including the left uncinate fasciculus (see Aoki et al., 2013, for a review). This white matter pathway connects the anterior temporal lobe including amygdala and hippocampus with prefrontal areas and is of particular interest since it plays a crucial role in processing socio-affective information in typically developing (TD) participants as well as in individuals with psychopathology (e.g., Frey et al., 2000; Johnstone et al., 2007; Pehrs et al., 2015; Zuurbier et al., 2013). As a consequence, we sought to link difficulties in socio-affective domains in individuals with ASD to white matter tissue structure in the left uncinate fasciculus.

\subsection{Socio-affective deficits in autism spectrum disorder}

Social deficits in individuals with ASD include failure to initiate reciprocal social interactions, verbal and non-verbal communication difficulties, decreased sensitivity to social and emotional cues, and poor performance on Theory of Mind tasks. Social withdrawal, indifference and even avoidance of affection or physical contact, reduced eye contact, and decreased joint attention and facial responsiveness are also common (Rutgers et al., 2004).

In addition to the well-documented deficits in the social domain, there is increasing awareness that individuals with ASD often experience considerable emotional disturbances. Not only do they exhibit more negative and less positive emotions in comparison to TD controls (e.g., Ben Shalom et al., 2006; Capps et al., 1993; Kasari and Sigman, 1997; Samson et al., 2012, 
2014), they also experience more frequent symptoms of anxiety and depression, are more irritable, have more frequent meltdowns or tantrums, are more aggressive, and show increased self-injurious behavior (Dominick et al., 2007; Gotham et al., 2013; Mazefsky et al., 2013; Quek et al., 2012; Vasa et al., 2013; White et al., 2009).

Recent studies have corroborated the critical role of emotion dysregulation in affective functioning (Mazefsky et al., 2013; Samson et al., 2012; Samson et al., 2015b). Individuals with ASD, compared to TD participants, seem to have a dysfunctional pattern of emotion regulation, characterized by less frequent use of adaptive emotion regulation strategies (goal-directed behaviors, seeking social support, cognitive reappraisal; Jahromi et al., 2012; Rieffe et al., 2011; Samson et al., 2012, 2015ac) and more frequent use of maladaptive strategies (avoidance, venting, expressive suppression; Jahromi et al., 2012; Konstantareas and Stewart, 2006; Samson et al., 2012, 2015a; Mazefsky et al., 2014). Emotion dysregulation in ASD has been linked to increased psychopathology (Mazefsky et al., 2014), lower levels of prosocial peer engagement (Jahromi et al., 2013) and higher levels of maladaptive behavior (Samson et al., 2015b).

The current study focuses on both social deficits and emotion regulation deficits because these socio-affective deficits are (1) heavily linked and thought to influence one another (emotion regulation often takes place in social contexts and is acquired through social interactions, e.g. between child and caregiver in early stages of life, see English et al., 2012; Shefield Morris et al., 2007), (2) evident in ASD, and finally, (3) are thought to rely on heavily overlapping neural structures.

\subsection{Potential neural correlates of socio-affective deficits in autism spectrum disorder}

In order to process and regulate socio-affective information, several brain regions need to work in concert. However, in ASD, neural connectivity between functionally connected regions 
seems to be altered (e.g., Aoki et al., 2013) which is in line with the aberrant connectivity hypothesis in ASD (Courchesne and Pierce, 2005). One particular promising candidate in explaining socio-affective deficits in ASD is the uncinate fasciculus.

The uncinate fasciculus connects polar temporal areas, including the amygdala and hippocampus, to cortical regions such as orbitofrontal cortex (OFC) (Kier et al., 2004; Olson et al., 2015; Von der Heide et al., 2013). It therefore connects basic emotion processing areas with regions involved in higher order processing, including emotion regulation. The uncinate has been linked to processing of emotional information in healthy populations (Frey et al., 2000), and has been reported to be related to impaired socio-emotional processing in disorders such as frontotemporal dementia (Von der Heide et al., 2013), and also more generally with symptom severity in individuals with ASD (Poustka et al., 2012).

While multiple white matter structures have been found to be affected in ASD (for a review, see Aoki et al., 2013; Barnea-Golary et al., 2004; Barnea-Golary et al., 2010; Olson et al., 2015), the left uncinate may be particularly important in processing socio-affective information (Pehrs et al., 2015; Olson et al., 2015). There seems to be a relatively left dominant abnormality in the uncinate fasciculus in ASD. Brain asymmetry usually occurs in typical brain maturation, which seems to be essential for emotion processing and other functions including cognition, sensory and motor functions. Atypical brain maturation in ASD and possible pathophysiology of disturbances of these functions may specifically affect the left uncinate (see Aoki et al. 2013). In the same lines, there is recent evidence that white matter tissue structure specifically in the left uncinate fasciculus is related to emotion regulation in a healthy population (Zuurbier et al., 2013). Furthermore, there is evidence that neurotypicals engage the left prefrontal cortex to down-regulate amygdala responses to negative stimuli. In contrast, patients 
with major depression, who have more difficulties regulating their emotions, do not seem to engage the left lateral-ventromedial prefrontal circuitry important for the down-regulation of amygdala responses (Johnstone et al., 2007). These findings suggest the importance of the involvement of the left uncinate in socio-affective skills including emotion regulation.

\subsection{The current study}

The goal of this study was to examine the role of one white matter structure, the left uncinate, in socio-affective processing in individuals with ASD compared to TD participants. We focused on children and adolescents, since socio-affective skills mature during this important developmental stage (Riediger and Klipker, 2014). Parent reports were used to assess socioaffective skills and clinical symptomatology given difficulties of accessing own mental (including emotional) states in individuals with ASD (Fitzgerald and Bellgrove, 2006).

We used Diffusion Tensor Imaging (DTI) to examine the tissue structure of white matter tracts implicated in processing of socio-emotional information. DTI allows for in-vivo measurement of fiber tracts in the brain. Diffusion properties are affected by biological properties of the axons (e.g. myelination, axonal density, and diameter) that are driven by overall white matter maturation, as well as geometric configurations of the axons (tract curvature, directional coherence, crossing, branching and merging fiber bundles). Fractional anisotropy (FA) is one of several diffusion parameters quantifying directionality of water diffusion on a scale from zero (equal diffusion in all directions) to one (diffusion in one direction, see Ameis et al., 2011) and reflects myelination, white matter organization, and the density of white matter tracts (Olson et al., 2015). Abnormal development of white matter may result in atypical white matter structure, which in turn may affect behavior, including socio-affective deficits. 
We expected individuals with ASD to have more socio-affective deficits than TD participants. Specifically, we expected individuals with ASD to have significantly more social deficits and lower control over their emotions compared to TD participants. Moreover, based on previous studies (Aoki et al., 2013, for a review), we hypothesized that individuals with ASD would have lower FA in the left uncinate. Furthermore, we expected group status to moderate the association between abnormal white matter structure in the left uncinate and socio-affective deficits. In particular, we expected white matter structure in the left uncinate to be linked to socio-affective skills in individuals with ASD, but not in TD individuals, since brain behavior associations are more frequently identified in individuals with neuropsychiatric disorders when compared to neurotypicals (Baur et al., 2011; Poustka et al., 2011; Thomason \& Thompson, 2011). To test specificity of group effects as well as moderating effects, we also analyzed group differences in the right uncinate fasciculus and tested moderation effects of group status on the association between white matter tissue structure in the uncinate fasciculi and another core feature of ASD, namely restricted and repetitive behaviors. We did not expect group differences or moderation effects related to the right uncinate, or repetitive behaviors.

\section{Methods}

\subsection{Participants}

Eighteen individuals with ASD (2 female) and 18 TD individuals ( 3 female) between the ages of 8 and 20 years participated in the study (see Table 1). The two groups did not differ in age or gender. The sample consisted of 25 Caucasians, 5 Asians, 2 Hispanics, 1 Afro-American, and 3 other ethnicities. Participants with ASD were recruited from a clinic specializing in ASD and developmental disabilities as well as from a research registry. TD participants were recruited through flyers advertising an emotion regulation study in areas that were comparable to the 
socio-economic status of the participants with ASD. Participants were enrolled as part of a larger project.

-----Insert Table 1 about here----

\subsection{Clinical assessment}

For participants with ASD, diagnosis was established through expert clinical evaluation (J.M.P. and A.Y.H.) based on the DSM-IV-TR (APA, 2000) and confirmed with the Autism Diagnostic Interview-Revised (ADI-R) and Autism Diagnostic Observation Schedule (ADOS; Lord et al., 1994, 2000). Two included individuals missed the cut off point on ADOS by one point ${ }^{1}$. Individuals with secondary autism related to a specific etiology (e.g., tuberous sclerosis, Fragile X) and individuals with evidence of genetic, metabolic, or infectious disorders were excluded.

TD participants were screened using face-to-face evaluations (Kiddie-Schedule for Affective Disorders and Schizophrenia for School-Aged Children, Kaufman et al., 1997), telephone interviews, and observation during psychometric tests. Exclusion of TD participants was also performed based on medical and psychiatric history.

Cognitive functioning (Full scale Intelligence quotient, FSIQ) was assessed using the Stanford Binet (5th edition, SB5, Roid, 2003). The ASD core features such as social and communication deficits and restricted and repetitive behavior were assessed with the Social Responsiveness Scale (SRS, Constantino and Gruber, 2005; Constantino et al., 2000) and the Repetitive Behavior Scale-Revised (RBS-R, Lam and Aman, 2007). Externalizing and internalizing behavior was assessed with the Child Behavior Checklist (Achenbach, 1991). SRS was available for 16 individuals with ASD and 16 TD participants, RBS-R was available for 17 
individuals with ASD and 16 TD participants, while CBCL was available for 17 individuals with ASD and 17 TD participants.

This study was approved by the University’s Institutional Review Board. Written informed consent was obtained from parents and assent from all participants.

\subsection{Emotion regulation assessment}

The items of the Self-Report Emotional Ability Scale (SEAS; Freudenthaler and Neubauer, 2005) were reformulated for the parents to report on their children's emotional control. One subscale of the SEAS was used in this study: Regulation of one 's own emotions (6 items, alpha $=.90$, item example: 'After an upsetting event, it is difficult for my child to calm down'). Responses are scored on a six-point scale ranging from 'not true' to 'very true'. The scale has already been used in several other recent studies (Freudenthaler and Neubauer, 2005; Freudenthaler et al., 2008; Papousek et al., 2008). Data on this questionnaire was available for 16 participants with ASD, and 16 TD participants. For the purpose of this study we reverse-scored the items to represent emotion regulation deficits rather than the ability to regulate emotions.

\subsection{Diffusion Tensor Imaging}

MR measurements were performed using a 3T GE Discovery MR750 MRI scanner and a 32-channel head coil from Nova Medical. High-resolution T1-weighted anatomical images were collected for each subject using an 8-minute sagittal IR-prep 3D fast SPGR sequence (1x1x1 mm voxel size). Diffusion measurements were performed using a diffusion-weighted dual-spin-echo EPI sequence with $2 \mathrm{~mm}$ isotropic resolution. We measured 30 non-collinear diffusion directions with a b-value of $1200 \mathrm{~s} / \mathrm{mm} 2$, plus three measurements with no diffusion weighting. This 5minute diffusion scan was repeated and all 66 measurements were used for tensor fitting. Head motion in the scanner was restricted by the use of extra padding around the head. Subject motion 
in the diffusion-weighted images was corrected by a rigid-body co-registration of each diffusionweighted image to the mean of the non-diffusion-weighted images with a two-stage coarse-tofine approach that maximizes the normalized mutual information. The two groups did not differ in their mean displacement $\left(M_{T D}=.83, S D=.76, M_{A S D}=.81, S D=.40, t(34)=.12, n s\right)$. There were also no correlations between mean displacement and FA in any of the 10 nodes of the right or left uncinate fasciculi ${ }^{2}$.

The Automated Fiber Quantification (AFQ, Yeatman et al., 2012) software package was used to identify 18 fiber tracts in each participant's brain and quantify tissue properties along the tract trajectory (for the purpose of this study we focused on the uncinate fasciculi only). AFQ uses a three-step procedure to identify each tract from an individual's diffusion data.

Deterministic streamlines tracking was used to estimate candidate pathways for the uncinate fasciculi. This was followed by a refinement step which compares each candidate streamline to a fiber tract probability map and removes candidates that pass through regions of the white matter that are unlikely to be part of the tract. Finally, the aggregate tract is summarized by a core fiber that follows the central position of all the tract fibers. This core fiber is used to define 10 samplepoints along each tract. Tissue properties are calculated along the trajectory of the core fiber by resampling to 10 equally spaced nodes, interpolating the tissue metric from the diffusion data within the tract, and calculating a weighted sum of each voxel associated with a given node based on its Mahalanobis distance from the core of the tract. The result is a vector of 10 measurements of each diffusion parameter sampled equidistantly along the trajectory of each fascicle.

\subsection{Statistical analyses}

Group differences in socio-affective deficits as well as repetitive behavior (a control 
variable) were computed with ANCOVAS using FSIQ as a covariate because of significant group differences in cognitive functioning ${ }^{3}$ (when FSIQ was included as covariate, $\mathrm{z}$ transformed FSIQ was used). For the analysis of the FA, we first computed a 2 x 2 repeated measures ANCOVA with the 2 hemispheres as repeated factor, with group as between subject factor, and with FSIQ as a covariate. This was followed by $2 \times 2$ repeated measurement ANCOVAs with the 2 hemispheres as repeated factor, with group as between subject factor, and with FSIQ as a covariate for each of the 10 nodes. Our main focus was on significant group $\mathrm{x}$ hemisphere interactions, which were followed up with ANCOVAs controlling for FSIQ to identify group differences in a specific node for the right and left hemisphere. To test whether group moderated the relationship between a specific node of the right or left uncinate (in which we had identified a significant group difference at $p<.01)$ and social deficits, emotion regulation deficits, and restricted and repetitive behaviors, we performed multiple regressions with three predictors (group, node, group x node) and one covariate (FSIQ). Group and nodes 5 and 6 in the left uncinate were highly correlated $(r s=-.51, p<.01)$. To handle multi-collinearity, we orthogonalized FA and group and used the residuals (with group partialled out) in the moderation analysis. Orthogonalizing resulted in acceptable low variance inflation factor (VIF) factors (all VIF's <2).

\section{Results}

\subsection{Preliminary analyses}

Individuals with ASD had lower FSIQ, and, as expected, exhibited more restricted and repetitive behaviors, had more social and communication deficits, and showed more psychopathology (externalizing and internalizing behavior, affective problems, and anxiety problems) compared to TD participants, suggesting significant emotional difficulties in the ASD 
group (see Table 1). Partial correlations controlling for FSIQ showed that social deficits and restricted and repetitive behaviors were highly correlated $(r(28)=.79, p<.001)$, and emotion regulation was linked to social deficits $(r(26)=.72, p<.001)$ and restricted and repetitive behaviors $(r(27)=.43, p<.001)$. Considering the ASD group only, partial correlations controlling for FSIQ revealed that the core features were still highly correlated $(r(13)=.76, p<.001)$, but emotion regulation was not correlated with social deficits $(r(12)=.19, n s)$ or restricted and repetitive behaviors $(r(12)=.05, \mathrm{~ns})$.

\subsection{Group differences in socio-affective deficits}

Individuals with ASD had more social deficits $\left(F(2,31)=36.20, p<.001, \eta^{2}=.71\right.$, as assessed with the SRS) and more difficulties regulating their own emotions $(F(2,31)=19.09$, $p<.001, \eta^{2}=.57$, see Table 1 for descriptive statistics). Controlling for FSIQ as a covariate did not affect any of these measures.

\subsection{Group differences in fractional anisotropy in the left and right uncinate fasciculi}

In order to examine whether there was a group by hemisphere interaction in general, a $2 \mathrm{x}$ 2 mixed model repeated measures ANCOVA with the 2 hemispheres as repeated factor, group as between subject factor, and with FSIQ as a covariate revealed a significant interaction between hemisphere and group $\left(F(1,33)=8.80, p<.01, \eta^{2}=.21\right)$. Differences were analyzed in each hemisphere separately, and differences between the two groups were found in the left $\left(M_{A S D}=.44\right.$, $\left.S D=.03, M_{T D}=.47, S D=.03 ; F(2,35)=6.02, p<.01, \eta^{2}=.27\right)$, and the right hemispheres $\left(M_{A S D}=.45\right.$, $\left.S D=.03, M_{T D}=.46, S D=.02 ; F(2,35)=8.04, p<.01, \eta^{2}=.33\right)$. The effect of FSIQ was also significant $\left(F(1,33)=6.67, p<.05, \eta^{2}=.17\right)$, but there was no main effect of the hemisphere $\left(F(1,33)=.26, \mathrm{~ns}, \eta^{2}=.01\right)$ or group effect overall $\left(F(1,33)=2.89, \mathrm{~ns}, \eta^{2}=.08\right)$. See Figure 1 for an illustration of the left and right uncinate in the two participants, one from each group. 
In a second step, 2 (hemispheres, repeated) x 2 (group) mixed model repeated measures ANCOVAs controlling for FSIQ were performed for each node. The following effects could be identified in nodes 5, 6, and 8 (see Figure 2): For node 5, we identified a significant interaction between hemisphere and group $\left(F(1,33)=9.43, \mathrm{p}<.01, \eta^{2}=.22\right)$. When group differences were analyzed in each hemisphere separately, group differences in the left $(F(2,35)=6.82, p<.01$, $\left.\eta^{2}=.29\right)$, and right hemispheres $\left(F(2,35)=7.54, p<.01, \eta^{2}=.31\right)$ were observed. The effect of FSIQ was also significant $\left(F(1,33)=5.32, p<.05, \eta^{2}=.14\right)$, but no significant effect of hemisphere $\left(F(1,33)=.83, \mathrm{~ns}, \eta^{2}=.02\right)$ or group $\left(F(1,33)=2.59, \mathrm{~ns}, \eta^{2}=.07\right)$ could be identified.

For node 6, we observed a significant interaction between hemisphere and group $\left(F(1,33)=9.12, \mathrm{p}<.01, \eta^{2}=.22\right)$. If group differences are analyzed in each hemisphere separately, we identified a group difference in the left $\left(F(2,35)=5.83, p<.01, \eta^{2}=.26\right)$, and right hemispheres $\left(F(2,35)=5.02, p<.05, \eta^{2}=.23\right)$. The effect of FSIQ was also significant $(F(1,33)=6.80, p<.05$, $\left.\eta^{2}=.17\right)$, as well as the group effect $\left(F(1,33)=5.08, \mathrm{~ns}, \eta^{2}=.13\right)$, but no significant effect of hemisphere $\left(F(1,33)=.55\right.$, ns, $\left.\eta^{2}=.02\right)$ could be identified.

For node 8, we identified a significant interaction between hemisphere and group $\left(F(1,33)=5.59, p<.05, \eta^{2}=.15\right)$. When group differences were analyzed in each hemisphere separately, there was no group difference in the left $\left(F(2,35)=1.35\right.$, ns, $\left.\eta^{2}=.08\right)$, but there was a difference in the right hemisphere $\left(F(2,35)=3.59, p<.05, \eta^{2}=.18\right)$. The effect of hemisphere $\left(F(1,33)=31.56, p<.001, \eta^{2}=.49\right)$ indicated higher FA in the left $(M=.56, S D=.06)$ compared to the right uncinate $(M=.51, S D=.06)$. The interaction with FSIQ was also significant $\left(F(1,33)=8.15, p<.05, \eta^{2}=.20\right)$, but no group effect $\left(F(1,33)=.29\right.$, ns, $\left.\eta^{2}=.01\right)$ could be identified ---Insert Figure 1 and 2 about here---

\subsection{White matter structure in the left uncinate and socio-affective deficits}


We next examined whether nodes with significant group differences on a level of $p<.01$ in each hemisphere (node 5 in the left and right uncinate, and node 6 in the left uncinate) were related to socio-affective deficits and / or restricted and repetitive behavior (control measure) in each of the two participant groups. In order to determine whether group moderated the relationship between FA and socio-affective deficits and / or restricted and repetitive behavior, we computed regression analyses with the interaction term uncinate $\mathrm{x}$ group (see Table 2 and Figure 3).

Social Deficits: For node 5 in both hemispheres, only group effects (no moderation effects) were observed using moderation analyses (see Table 2). However, in node 6 of the left uncinate, a moderator effect was observed $(\beta=-.23, p<.05, d=.73)$; lower FA in this specific node was associated with higher social deficits in individuals with ASD ( $t=-3.01, p=.006)$ but not TD $(t=-0.613, p=.55$; see Figure 3).

Emotion Regulation Deficits: For node 5 in both hemispheres, only group effects (no moderation effects) were observed using moderation analyses (see Table 2). For the node 6 of the left uncinate, however, a moderator effect was observed $(\beta=-.28, p=.05, d=.72)$, lower FA in this specific node was associated with higher emotion regulation deficits in individuals with $\operatorname{ASD}(t=-2.64, p=.01)$ but not TD $(t=-0.34, p=.73$, see Figure 3$)$.

Repetitive Behavior: As a control analysis, we also assessed the moderating effect of group on the association between FA in the left uncinate and repetitive behavior. Using moderation analyses, only group effects were identified for nodes 5 in both hemispheres and node 6 of the left uncinate (see Table 2).

To summarize the findings of these moderation analyses, we found that group moderated the effect in one node of the left uncinate for both social deficits and emotion regulation deficits, 
but not repetitive behavior, indicating that FA in this white matter region was associated with socio-affective deficits in individuals with ASD.

---Insert Table 2 and Figure 3 about here---

\section{Discussion}

In the current study, we examined the relationship between trait socio-affective deficits and structural OFC-amygdala connectivity in ASD. By means of DTI, we measured FA in the uncinate fasciculus bilaterally, to better understand the structural connections between prefrontal cortex and amygdala and other structures, including the hippocampus. The current study revealed and replicated group differences in socio-affective deficits (see Constantino et al., 2000; Mazefsky et al., 2014; Rieffe et al., 2011; Samson et al., 2012, 2014; White et al., 2009). More importantly, we identified lower FA in the left uncinate and, contrary to our expectations, at times also in the right uncinate. This latter observation seems to be in contrast to some previous studies reporting on more pronounced group differences in the left uncinate (see Aoki et al., 2013, for a review). The difference between the left and right uncinate in ASD, is intriguing and warrants further investigation.

Additionally, our study provided preliminary evidence that white matter characteristics of the left uncinate are specifically linked to socio-affective deficits in the ASD group, highlighting the importance of connectivity between the amygdala and prefrontal areas for social skills and emotion regulation which may be a cause or result of aberrant functioning. The present study corroborates aberrant connectivity in ASD (Belmonte, Allen, Beckel-Mitchener, Boulanger et al., 2004; Courchesne and Pierce, 2005; Vissers et al., 2012) and highlights the relationship between the left uncinante and socio-affective deficits. 
Lower FA in the uncinate may point to a dysfunctional interaction between the orbitofrontal-centered and hippocampal affect systems as defined in the recently published Quartet Theory on Emotions (Koelsch et al., 2015). The theory suggests that four different affect systems in the brain generate different classes of affect. The hippocampal system is involved in the generation of positive attachment-related emotions, while the OFC-system performs an automatic appraisal of external and internal information and is involved in regulating emotions. Pehrs et al. (2015) put forward the hypothesis that not only the OFC - amygdala connectivity may be relevant to socio-affective processes in individuals with ASD, but also the connection between OFC and hippocampus. Von der Heide et al. (2013) suggested that the uncinate fasciculus is involved in how stored information alters behavior such as approaching or avoiding stimuli based on reward or punishment history. Therefore, difficulties in developing emotional attachments and friendships and the ability to learn from social praise and other rewards in the social context could potentially be linked to dysfunctions in connectivity between the OFC hippocampal affect systems, mediated by the uncinate fasciculus (see also Olson et al., 2015). While the Quartet Theory may provide a good model to understand socio-affective deficits in individuals with ASD, its validity to help explain such deficits warrants further examination.

As expected, restricted and repetitive behaviors were not related to FA in the left uncinate fasciculus in individuals with ASD. This highlights the specificity of our findings regarding the relationship between the left uncinate structure and socio-affective deficits. Although repetitive behaviors have been shown to be related to emotion dysregulation (Samson et al., 2014) and may at times be used to regulate emotions (Samson et al., 2015c), there is no evidence from the current study that repetitive behavior is linked with the connectivity between basic (e.g., amygdala) and higher order emotion processing areas (e.g., OFC). This suggests that repetitive 
behaviors, when used to regulate emotions, might involve different pathways in the brain. However, more research is needed to better understand repetitive behaviors in relation to emotion regulation.

Associations between the left uncinate and socio-affective skills were only found in the ASD group and not in typically developing participants. There seems to be a tendency for associations between trait measures and abnormal white matter structure to be found in clinical populations rather than in healthy controls. For example, Beyer et al. (2014) did not find an association between trait anger or physical aggressiveness and FA in bilateral uncinate in healthy participants. In contrast, deficits in structural prefrontal-amygdala connectivity were identified in criminal offenders with psychopathy and adolescents and adults with conduct disorder. This may be related to higher variability in the assessed trait in clinical groups. However, in the current study, we identified higher variability only in social deficits as revealed by Levene's tests $(F=8.90, p<.01)$, but not for emotion regulation or FA in node 6 of the left uncinate. In addition, DTI studies in individuals with affective disorders consistently identify lower FA in frontal and temporal lobes and tracts (Sexton et al., 2009). Nevertheless, studies of healthy participants also found associations between FA-values in left uncinate fasciculus and individual trait measures such as anxiety (Montag et al., 2012) and emotion regulation (Zuurbier et al., 2013).

\subsection{Limitations and future directions}

Using DTI, our findings shed new light on the association between socio-affective deficits and FA in the left uncinate fasciculus. However, it is also important to mention several methodological limitations of the present study.

First, we studied a relatively small number of individuals with ASD and TD controls. This may have led to being underpowered to find moderation effects in several nodes of the left 
uncinate. It is possible that a bigger sample may have led to more robust effects and detectable associations in other nodes of this white matter structure. Therefore, the current results need to be replicated in larger samples.

Second, we focused on parents' reports of socio-affective deficits in children and adolescents with ASD. Future studies should include larger samples while using self-report as well as behavioral measures of social deficits and emotion regulation.

Finally, it is known that the uncinate fasciculus is one of the latest developing tracts with its maturational peak in the third decade of life (Olson et al., 2015). This makes it a potentially interesting structure to better understand in longitudinal designs treatment effectiveness in the domain of socio-affective skills in psychopathology.

\subsection{Conclusions}

To the best of our knowledge, this is the first study to link socio-affective deficits in ASD to abnormal white matter tissue structure in the left uncinate. Evidence of links between emotion regulation difficulties in ASD and reduced prefrontal-amygdalar connectivity has started to emerge (Pitskel et al., 2014), but additional comprehensive studies replicating differences in structural and functional connectivity between prefrontal and limbic areas in the brains of individuals with ASD - as well as links to socio-affective deficits - are still needed. 


\section{Funding}

The research project was supported by the Swiss National Science Foundation PA00P1_136380 (A.S.), by the Stanford Center for Cognitive and Neurobiological Imaging and by the Mosbacher Family Fund for Autism Research.

Conflicts of Interests: None of the authors has any conflicts of interest.

\section{Acknowledgements}

The authors would like to thank Yasmin Arbab, Yael Enav, and Shweta Shah, for their help conducting this study. We would like to thank Naama Barnea-Goraly for comments on a previous version of this manuscript.

\section{Contributors}

A.C.S., J.J.G., and A.Y.H. planned the study, A.C.S. conducted the study, J.M.P and A.Y.H. were responsible for and monitored the clinical assessments, R.F.D. and A.C.S. analyzed the neuroimaging data, A.C.S. and I.A.L. were responsible for the statistical analysis of the data, all authors contributed substantially to writing the article and approved the manuscript. 


\section{Footnotes}

1 Despite the small sample size, we also ran all analyses with females excluded, each borderline participant, or both, females and borderline participants. All results remained consistent, except the moderation effects of emotion regulation dropped in some analyses to trend level (e.g., $p=.067$, when excluding all females and both borderline participants, $\mathrm{N}=7$ ). Therefore, we decided to include all participants for the analyses.

2 Since one TD participant had high levels of movement, we re-ran all the analyses after excluding this participant. All of the significant results in all analyses remained consistent, except the moderation effect of emotion regulation in node 6 of the left uncinate, which dropped to trend level ( $p=.069)$. We also included the absolute root mean square of movement as additional covariate of nuisance, which did not affect most of the results except of the moderation effects, which dropped also slightly to trend level. This may be due to too many predictors included for such a small sample size.

3 Analyses were also conducted with age as an additional covariate due to the broad age range in our study. Including age as covariate did not change any of the results except the moderation analysis for emotion regulation (the moderation effect was marginal, $p=.058$ ). However, we decided to only control for FSIQ in the main text because the two groups did not differ in age and our sample size was small. 


\section{References}

Achenbach, T.M. 1991. Integrative guide to the 1991 CBCL/4-18, YSR, and TRF profiles, Burlington, VT, University of Vermont, Department of Psychology.

Ameis, S.H., Fan, J., Rockel, C., Voineskos, A.N., Lobaugh, N.J., Soorya, L., Wang, A.T., Hollander, E., Anagnostou, E. 2011. Impaired structural connectivity of socio-emotional circuits in autism spectrum disorders: A diffusion tensor imaging study. PLoS one. 6, e28044.

American Psychiatric Association. 2000. Diagnostic and statistical manual of mental disorders, fourth ed. text rev. Washington, DC, Author.

American Psychiatric Association. 2013. Diagnostic and statistical manual of mental disorders: DSM-5, Washington, DC, Author.

Aoki, Y. Abe, O., Nippashi, Y., Yamasue, H., 2013. Comparison of white matter integrity between autism spectrum disorder subjects and typically developing individuals: a metaanalysis of diffusion tensor imaging tractography studies. Mol. Autism. 4, 25.

Barnea-Golary, Lotspeich, L., Reiss, A. A. 2010. Similar white matter aberrations in children with autism and their unaffected siblings. Arch. Gen. Psychiatry. 67, 1052-1060.

Barnea-Golary, N., Kwon, H., Menon, V., Eliez, S., Lotspeich, L., Reiss, A. A. 2004. White matter structure in autism: Preliminary evidence from Diffusion Tensor Imaging. Biol Psychiatry. 55, 323-326. Doi: 10.1016/j.biopsych.2003.10.022

Baur, V., Hänggi, J., Rufer, M., Delsignore, A., Jäncke, L., Herwig, U., Brühl, A. B. 2011. White matter alterations in social anxiety disorder. J. Psychiatr. Res. 45, 1366-1372. 
Belmonte, M.K., Allen, G., Beckel-Mitchener, A., Boulanger, L.M., Carper, R.A., Webb, S.J. 2004. Autism and abnormal development of brain connectivity. J. Neurosci. 24, 92289231.

Ben Shalom, D., Mostofsky, S.H., Hazlett, R.L., Goldberg, M.C., Landa, R.J., Faran, Y., . . . Hoehn-Saric, R. 2006. Normal physiological emotions but differences in expression of conscious feelings in children with high-functioning autism. J. Autism. Dev. Disord. 36, 395-400. doi:10.1007/s10803-006-0077-2

Beyer, F., Münte, T.F., Wiechert, J., Heldmann, M., Krämer, U.M., 2014. Trait aggressiveness is not related to structural connectivity between orbitofrontal cortex and amygdala. PLoS ONE. 9, e101105. doi:10.1371/journal.pone.0101105

Capps, L., Kasari, C., Yirmiya, N., Sigman, M., 1993. Parental perception of emotional expressiveness in children with autism. J. Consult. Clin. Psychol. 61, 475-484. doi:10.1037/0022-006X.61.3.475

Constantino, J.N., Przybeck, T., Friesen, D., Todd, R.D., 2000. Reciprocal social behavior in children with and without pervasive developmental disorders. J. Dev. Behav. Pediatr. 21, $2-11$.

Constantino, J.N., Gruber, C.P., 2005. Social responsiveness scale (SRS). Los Angeles, CA: Western Psychological Services.

Courchesne, E., Pierce, K., 2005. Why the frontal cortex in autism might be talking only to itself: local overconnectivity but long-distance disconnection. Curr. Opin. Neurobiol. 15, 225-30. Dominick, K.C., Ornstein Davis, N., Lainhart, J., Tager-Flusberg, H., Folstein, S. 2007. Atypical behaviors in children with autism and children with a history of language impairment. Res. Dev. Disabil. 28, 145-162. 
English, T., John, O.P., Srivastava, S., Gross, J.J., 2012. Emotion regulation and peer-rated social functioning: A 4-year longitudinal study. J. Res. Pers. 46, 780-784. doi:10.1016/j.jrp.2012.09.006

Fitzgerald, M., Bellgrove, M.A., 2006. The overlap between alexithymia and Asperger's syndrome. J. Autism. Dev. Disord. 36, 573-57. doi:10.1007/s10803-006-0096-z

Freudenthaler, H.H., Neubauer, A.C., 2005. Emotional Intelligence: The convergent and discriminant validities of intra- and interpersonal emotional abilities. Pers. Individ. Dif. 39, $569-579$.

Freudenthaler, H.H., Neubauer, A.C., Gabler, P., Scherl, W.G., Rindermann, H. 2008. Testing and validating the trait emotional intelligence questionnaire (TEIQue) in a Germanspeaking sample. Pers. Indiv. Dif. 45, 673-678.

Frey, S., Kostopoulos, P., Petrides, M. 2000. Orbitofrontal involvement in the processing of unpleasant auditory information. Eur. J. Neurosci. 12, 3709-12.

Gotham, K., Bishop. S.L., Hus, V., Huerta, M., Lund, S., Buja, A., Krieger, A., Lord, C., 2013. Exploring the relationship between anxiety and insistence on sameness in Autism Spectrum Disorder, Autism. Res. 6, 33-41.

Von der Heide, R, Skipper, L.M., Klobusicky, E., Olson, I.R., 2013. Dissecting the uncinate fasciculus: disorders, controversies, and a hypothesis. Brain. 136, 1692-1707.

Jahromi, L.B., Meek, S.E., Ober-Reynolds, S., 2012. Emotion regulation in the context of frustration in children with high functioning autism and their typical peers. J. Child. Psychol. Psychiatry. 53, 1250-1258. DOI: 10.1111/j.1469-7610.2012.02560.x 
Jahromi, L.B., Bryce, C.I., Swanson, J., 2013. The importance of self-regulation for the school and peer engagement of children with high-functioning autism. Res. Autism. Spectr. Disord. 7, 235-246. http://dx.doi.org/10.1016/j.rasd.2012.08.012

Johnstone, T., van Reekum, C.M., Urry, H.L., Kalin, N.H., Davidson, R.J., 2007. Failure to regulate: Counterproductive recruitment of top-down prefrontal-subcortical circuitry in major depression. J. Neurosci, 27, 8877-8884; doi: 10.1523/JNEUROSCI.2063-07.2007

Kasari, C., Sigman, M., 1997. Linking parental perceptions to interactions in young children with autism. J. Autism. Dev. Disord. 27, 39-57. doi:10.1023/A:1025869105208

Kaufman, J., Birmaher, B., Brent, D., Rao, U., Flynn, C., Moreci, P., Williamson, D., Ryan, N., 1997. Schedule for affective disorders and schizophrenia for school-age children-present and lifetime version (K-SADS-PL): Initial reliability and validity data. J. Am. Acad. Child. Adolesc. Psychiatry. 36, 980-988.

Kier, E.G., Staib, L.H., Davis, L.M., Bronen, R.A., 2004. MR imaging of the temporal stem: anatomic dissection tractography of the uncinate fasciculus, inferior occipital fasciculus, and Meyer's loop of the optic radiation. AJNR. Am. J. Neuroradiol. 25, 677-691.

Koelsch, S., Jacobs, A., Menninghaus, W., Liebal, K., Klann-Delius, G., von Scheve, C., Gebauer, G., 2015. The Quartet Theory of Human Emotions: An integrative and neurofunctional model. Phys. Life. Rev. 13, 1-27. doi:10.1016/j.plrev.2015.03.001

Konstantareas, M., Stewart, K., 2006. Affect regulation and temperament in children with autism spectrum disorder. J. Autism. Dev. Disord. 36, 143-154. DOI 10.1007/s10803-005-0051-4 Lam, K.S., Aman, M.G., 2007. The Repetitive Behavior Scale-Revised: Independent validation in individuals with autism spectrum disorders. J. Autism. Dev. Disord. 37, 855-866. 
Laurent, A.C., Rubin, E. 2004. Challenges in emotional regulation in Asperger's Syndrome and High-Functioning Autism. Top. Lang. Disord. 24, 286-297.

Lord, C., Risi, S., Lambrecht, L., Cook, E.H., Jr., Leventhal, B.L., DiLavore, P.C., Pickles, A., et al., 2000. The autism diagnostic observation schedule-generic: A standard measure of social and communication deficits associated with the spectrum of autism. J. Autism. Dev. Disord. 30, 205-223.

Lord, C., Rutter, M., Le Couteur, A., 1994. Autism Diagnostic Interview-Revised: a revised version of a diagnostic interview for caregivers of individuals with possible pervasive developmental disorders. J. Autism. Dev. Disord. 24, 659-685.

Mazefsky, C.A., Herrington, J., Siegel, M., Scarpa, A., Maddox, B.B., Scahill, L., White, S.W., 2013. The role of emotion regulation in autism spectrum disorder. J. Am. Acad. Child. Adolesc. Psychiatry. 52, 679-688.

Mazefsky, C.A., Borue, Z., Day, T.N., Minshew, N.J., 2014. Emotion regulation patterns in adolescents with high-functioning autism spectrum disorder: Comparison to typically developing adolescents and association with psychiatric symptoms. Autism. Res. 7, 344354. DOI: 10.1002/aur.1366

Montag, C., Reuter, M., Weber, B., Markett, S., Schoene-Bake, J.C., 2012. Individual differences in trait anxiety are associated with white matter tract integrity in the left temporal lobe in healthy males but not females. J. Neurosci. 217, 77-83. http://dx.doi.org/10.1016/j.neuroscience.2012.05.017

Olson, I.R., Von der Heide, R.J., Alm, K.H., Vyas, G., 2015. Development of the uncinate fasciculus: Implications for theory and developmental disorders. Dev. Cogn. Neurosci. 14, $50-61$. 
Papousek, I., Freudenthaler, H., Schulter, G., 2008. The interplay of perceiving and regulating emotions in becoming infected with positive and negative moods. Pers. Individ. Dif. 45, $463-467$.

Pehrs, C., Samson, A.C., Gross, J.J., 2015. The Quartet Theory: Implications for autism spectrum disorder. Phys. Life. Rev. 13, 77-79.

Pitskel, N.B., Bolling, D.Z., Kaiser, M.D., Pelphrey, K.A., Crowley, M.J., 2014. Neural systems for cognitive reappraisal in children and adolescents with autism spectrum disorder. Dev. Cogn. Neurosci. 10, 117-28.

Poustka, L., Jennen-Steinmetz, C., Henze, R., Vomstein, K., Haffner, J., Sieltjes, B., 2012. Fronto-temporal disconnectivity and symptom severity in children with autism spectrum disorder. World. J. Biol. Psychiatry. 13, 269-280. doi:10.3109/15622975.2011.591824

Quek, L.-H., Sofronoff, K., Sheffield, J., White, A., Kelly, A., 2012. Co-occuring anger in young people with Asperger's syndrome. J. Clin. Psychol. 68, 1142-1148. DOI: $10.1002 /$ jclp. 21888

Riediger, M., Klipker, K., 2014. Emotion regulation in adolescence. in Gross, J.J. (Ed., $2^{\text {nd }}$ edition), Handbook of emotion regulation. Guilford Press, New York, pp. 3-24.

Rieffe, C., Oosterveld, P., Meerum Terwogt, M., Mootz, S., van Leeuwen, E., Stockmann, L., 2011. Emotion regulation and internalizing symptoms in children with autism spectrum disorders. Autism. 15, 655-670. DOI: 10.1177/1362361310366571

Roid, G.H. 2003. Stanford Binet's Intelligence Scales. Itasca, IL: Riverside Publishing. DOI: $10.1177 / 082957350401900113$ 
Rutgers, A.H., Bakermans-Kranenburg, M.J., van Ijzendoorn, M.H., van Berckelaer-Onnes, I.A., 2004. Autism and attachment: a meta-analytic review. J. Child. Psychol. Psychiatry. 45, 1123-34.

Samson, A.C., Huber, O., Gross, J.J., 2012. Emotion regulation in Asperger's syndrome and high functioning autism. Emotion. 12, 659-665. DOI: 10.1037/a0027975

Samson, A.C., Hardan, A.Y., Podell, R.W., Phillips, J.M., Gross, J.J., 2015a. Emotion regulation in children and adolescents with autism spectrum disorder. Autism. Res. 8, 9-18. doi: 10.1002/aur.1387

Samson, A.C., Hardan, A.Y., Lee, I.A., Phillips, J.M., Gross, J.J. 2015b. Maladaptive behavior in autism spectrum disorder: The role of emotion experience and emotion regulation. $\mathrm{J}$. Autism. Dev. Disord. 45, 3424-32. doi: 10.1007/s10803-015-2388-7.

Samson, A.C., Phillips, J.M., Parker, K.J., Shah, S., Gross, J.J., Hardan, A.Y. 2014. Emotion dysregulation and the core features of autism spectrum disorder. J. Autism. Dev. Disord. 44, 1766-1772. DOI 10.1007/s10803-013-2022-5

Samson, A.C., Wells, W.M., Phillips, J.M., Hardan, A.Y., Gross, J.J., 2015c. Emotion regulation in autism spectrum disorder: Evidence from parent interviews and children's daily diaries. J. Child. Psychol. Psychiatry. 56, 908-913. doi:10.1111/jcpp.12370

Shefield Morris, A., Silk, J.S., Steinberg, L., Myers, S.S., Robinson, L.R., 2007. The role of the family context in the development of emotion regulation. Soc. Dev. 16, 361-388. doi: 10.1111/j.1467-9507.2007.00389.x

Sexton, C.E., Mackay, C.E., Ebmeier, K.P., 2009. A systematic review of Diffusion Tensor Imaging Studies in affective disorders. Biol. Psychiatry. 66, 814-823. doi:10.1016/j.biopsych.2009.05.024 
Thomason M.E., Thompson, P.M., 2001. Diffusion imaging, white matter, and psychopathology. Annu. Rev. Clin. Psychol, 7, 63-85.

Vasa, R.A., Kalb, L., Mazurek, M., Kanne, S., Freedman, B., Keefer, A., Clemons, T., Murray, D., 2013. Age-related differences in the prevalence and correlates of anxiety in youth with autism spectrum disorders, Res. Autism. Spectr. Disor. 7, 1358-1369.

doi:10.1016/j.rasd.2013.07.005

Vissers, M.E., Cohen, M.X., Geurts, H.M., 2012. Brain connectivity and high functioning autism: a promising path of research that needs refined models, methodological convergence, and stronger behavioral links. Neurosci. Biobehav. Rev. 36, 604-625.

White, S.W., Oswald, D., Ollendick, T., Scahill, L., 2009. Anxiety in children and adolescents with autism spectrum disorders. Clin. Psychol. Rev. 29, 216-229. doi:10.1016/j.cpr.2009.01.003.

Yeatman, J.D., Dougherty, R.F., Myall, N.J., Wandell, B.A., Feldman, H.M., 2012. Tract Profiles of White Matter Properties: Automating Fiber-Tract Quantification. PLoS ONE. 7, e49790. doi:10.1371/journal.pone.0049790

Zuurbier, L.A., Nikolova, Y.S., Åhs, F., Hariri, A.R., 2013. Uncinate fasciculus fractional anisotropy correlates with typical use of reappraisal in women but not men. Emotion. 13, 385-90. doi: 10.1037/a0031163 
Table 1

Sample Characteristics

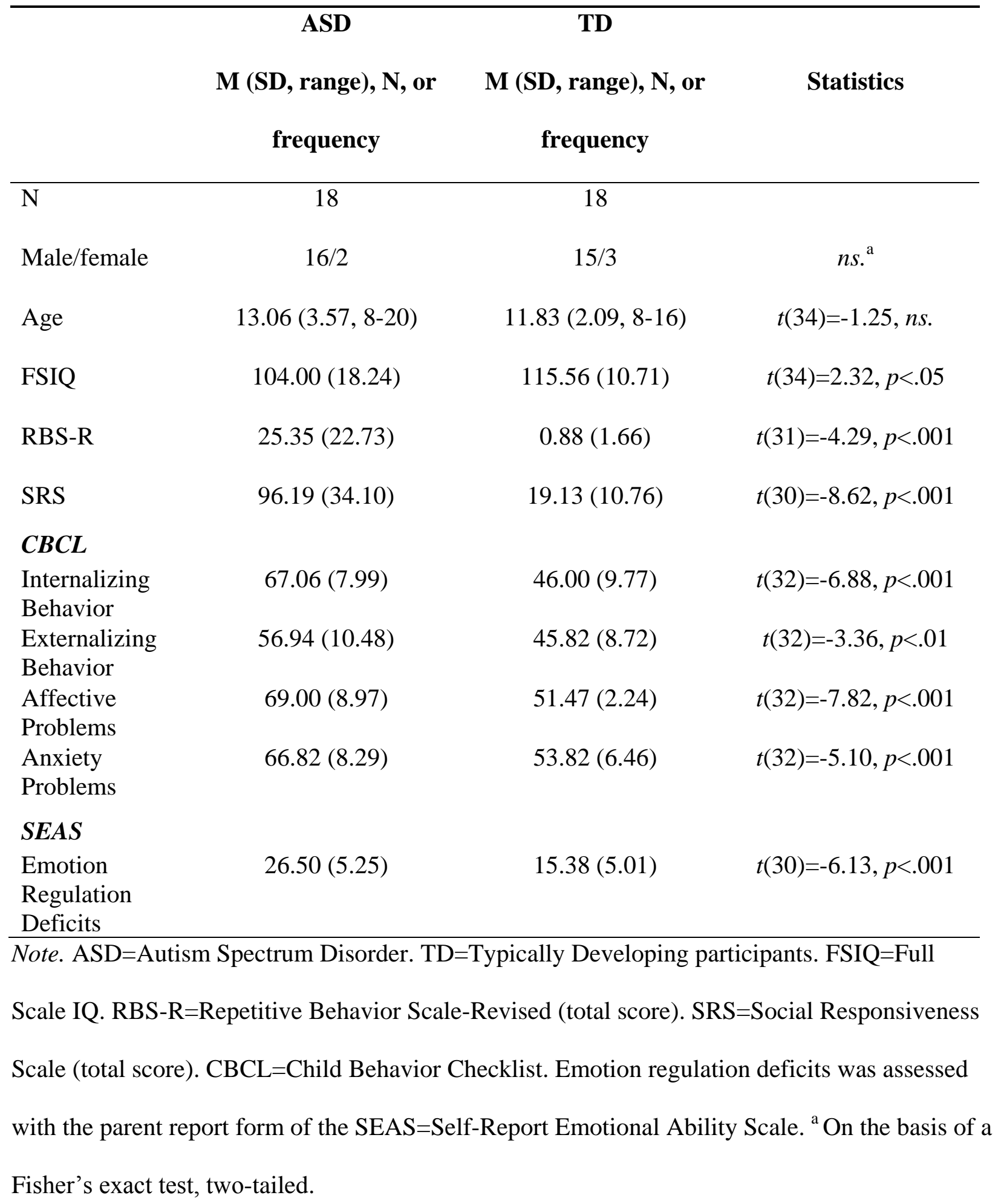


Table 2: Multiple regressions predicting social deficits, emotion regulation deficits, and restricted and repetitive behavior.

\begin{tabular}{|c|c|c|c|c|c|c|}
\hline & $\begin{array}{c}\text { Node } 5 \\
\text { right }\end{array}$ & & $\begin{array}{c}\text { Node } 5 \\
\text { left }\end{array}$ & & $\begin{array}{l}\text { Node } 6 \\
\text { ft uncin }\end{array}$ & \\
\hline & $\beta$ & $t(d f)$ & $\beta$ & $t(d f)$ & $\beta$ & $t(d f)$ \\
\hline Social Deficits (SRS) & & & & & & \\
\hline group & $.86^{* * *}$ & $7.70(\mathrm{df}=27)$ & $.86^{* * *}$ & $7.97(\mathrm{df}=27)$ & $.84 * * *$ & $8.99(\mathrm{df}=27)$ \\
\hline L uncinate (node) ${ }^{1}$ & -.01 & -.08 & -.027 & -.12 & -.07 & -.62 \\
\hline group x L uncinate $(\text { node })^{1}$ & .21 & .13 & -.06 & -.27 & $-.23 *$ & -2.07 \\
\hline FSIQ & .04 & .32 & .06 & .51 & -.005 & -.05 \\
\hline Emotion Regulation Deficits & & & & & & \\
\hline group & $.73 * * *$ & $5.32(\mathrm{df}=27)$ & $.73 * * *$ & $5.67(\mathrm{df}=27)$ & $.65 * * *$ & $5.23(\mathrm{df}=27)$ \\
\hline L uncinate (node) ${ }^{1}$ & .16 & 1.11 & .06 & .25 & -.05 & -.35 \\
\hline group $x$ L uncinate $(\text { node })^{1}$ & -.24 & -1.63 & -.32 & -1.35 & $-.28 *$ & -2.04 \\
\hline FSIQ & -.08 & -.52 & -.06 & -.47 & -.17 & -1.37 \\
\hline Restricted and Repetitive & & & & & & \\
\hline Behavior (RBS-R) & & & & & & \\
\hline group & $.56 * *$ & $3.47(\mathrm{df}=28)$ & $.56^{* * *}$ & $3.55(\mathrm{df}=28)$ & $.55 * * *$ & $3.58(\mathrm{df}=28)$ \\
\hline L uncinate $(\text { node })^{1}$ & .05 & .22 & .06 & .18 & .00 & .01 \\
\hline group $x$ L uncinate $(\text { node })^{1}$ & -.05 & -.21 & -.03 & -.11 & -.14 & -.78 \\
\hline FSIQ & -.15 & -.08 & -.15 & -.91 & -.17 & -1.05 \\
\hline
\end{tabular}

Notes. Node 5 (right uncinate): Social deficits: $\mathrm{R}^{2}=.71$, Adjusted $\mathrm{R}^{2}=.67, F(4,31)=16.87, p<.001$. Emotion regulation deficits: $\mathrm{R}^{2}=.78$, Adjusted $\mathrm{R}^{2}=.61, F(4,31)=10.56, p<.001$. Restricted and repetitive behavior: $\mathrm{R}^{2}=.39$, Adjusted $\mathrm{R}^{2}=.30, F(4,32)=4.49, p<.01$.

Node 5 (left uncinate): Social deficits: $\mathrm{R}^{2}=.72$, Adjusted $\mathrm{R}^{2}=.68, F(4,31)=17.42, p<.001$. Emotion regulation deficits: $\mathrm{R}^{2}=.64$, Adjusted $\mathrm{R}^{2}=.58, F(4,31)=11.86, p<.001$. Restricted and repetitive behavior: $\mathrm{R}^{2}=.39$, Adjusted $\mathrm{R}^{2}=.30, F(4,32)=4.49, p<.01$. Node 6 (left uncinate): Social deficits: $\mathrm{R}^{2}=.79$, Adjusted $\mathrm{R}^{2}=.76, F(4,31)=25.16, p<.001$. Emotion regulation deficits: $\mathrm{R}^{2}=.66$, Adjusted $\mathrm{R}^{2}=.61, F(4,31)=12.99, p<.001$. Restricted and repetitive behavior: $\mathrm{R}^{2}=.41$, Adjusted $\mathrm{R}^{2}=.33, F(4,32)=4.85, p<.01$. Standardized coefficients are reported; FSIQ=Full Scale IQ. RBS-R=Repetitive Behavior Scale-Revised (total score). SRS=Social Responsiveness Scale (total score). Emotion regulation was assessed with the parent report form of the SEAS=Self-Report Emotional Ability Scale.

1 Residual L uncinate (node), with group partialled out. 
Significant moderation effects are highlighted in bold. $* p<.05, * * p<.01, * * * p<.001$. 


\section{Figure Legends}

Figure 1. Left and right uncinate fasciculi for one individual with ASD (A) and one typically developing participant (B).

Figure 2. Group differences in the 10 nodes of the left and right uncinate fasciculi in individuals with Autism Spectrum Disorder (ASD) and typically developing (TD) participants.

Note. $* p<.05, * * p<.01$.

Figure 3. Interaction plots on the association between social deficits and emotion regulation deficits and FA in the left uncinate (node 6) in individuals with Autism Spectrum Disorder (ASD) and Typically Developing (TD) participants. 
A

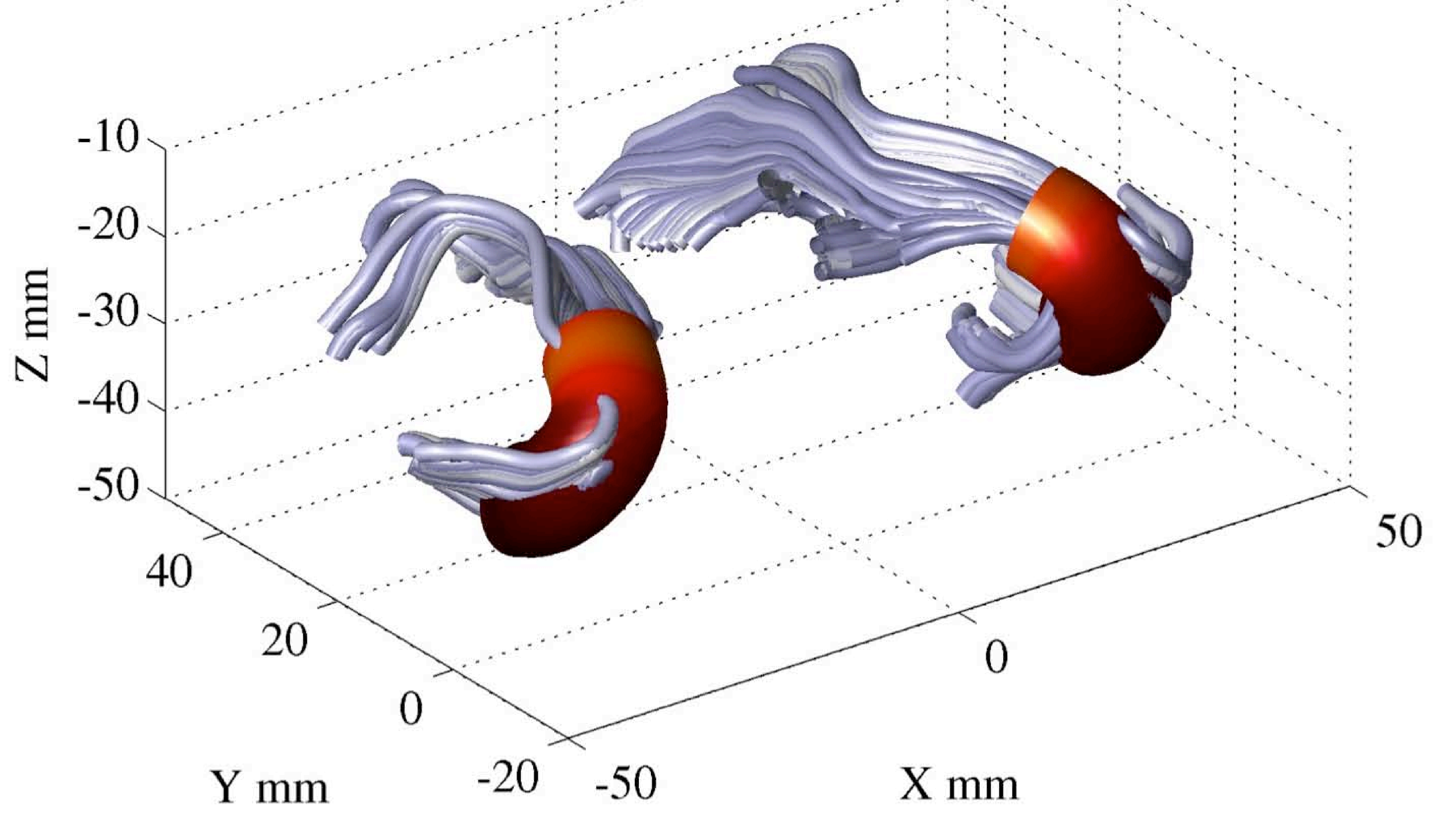

B

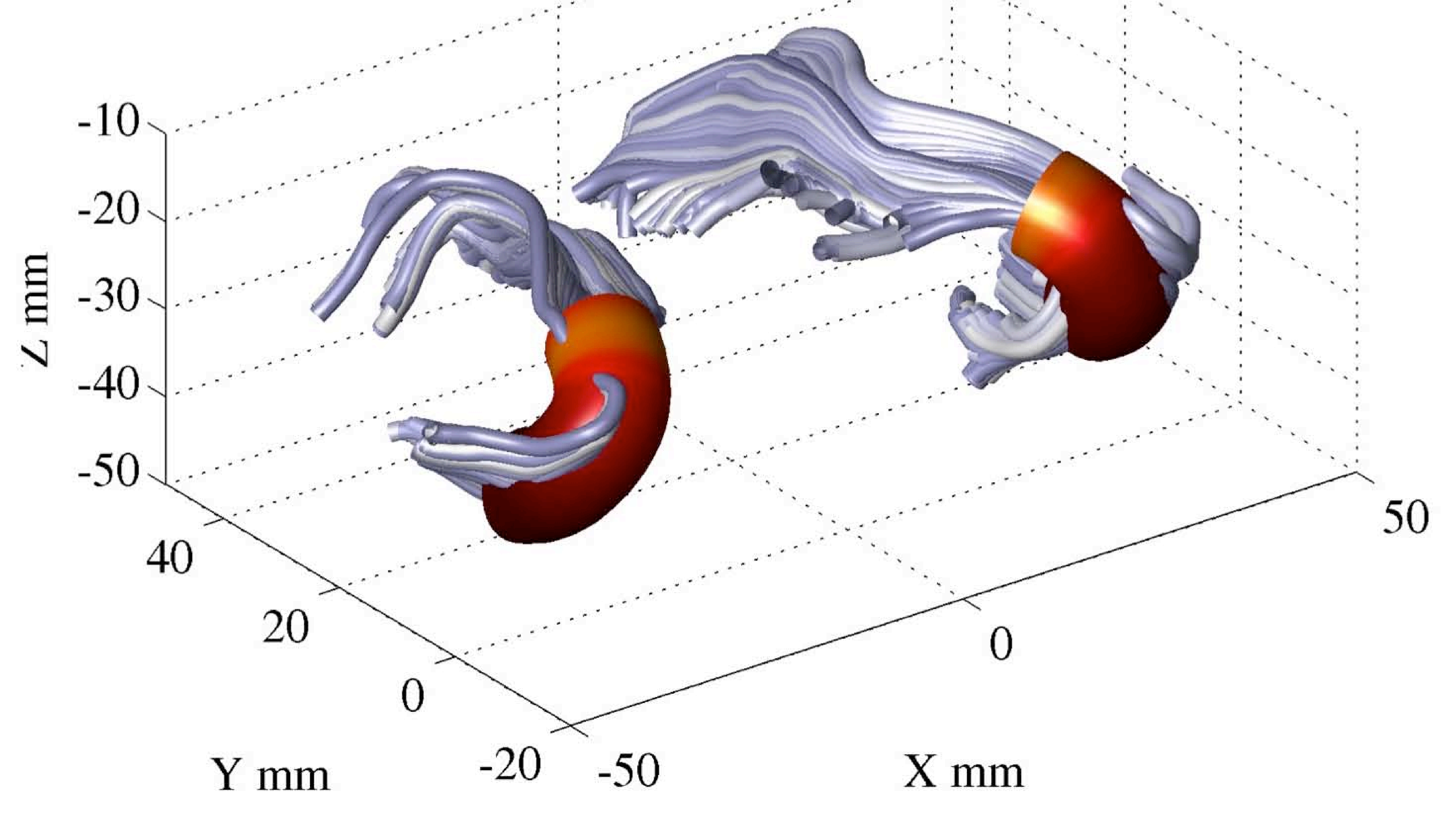

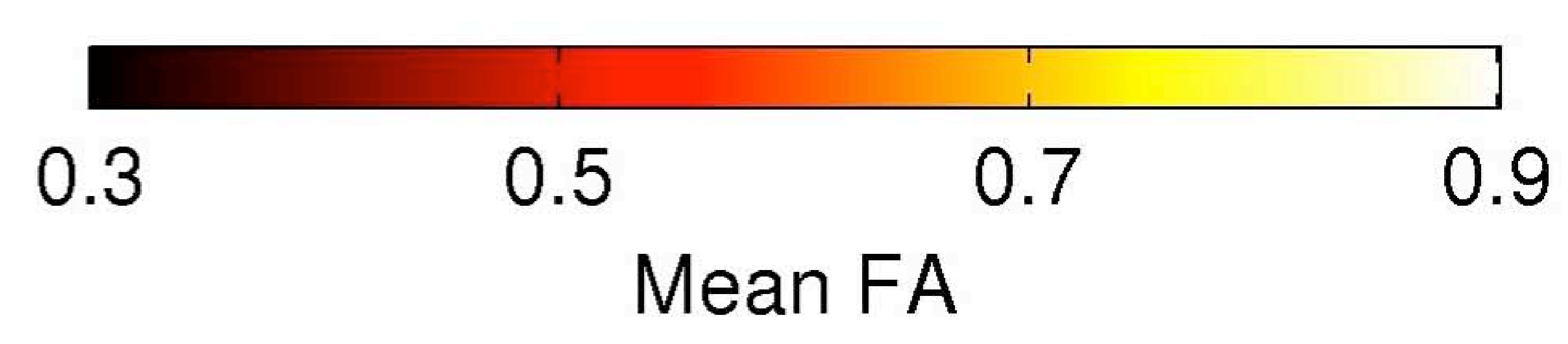



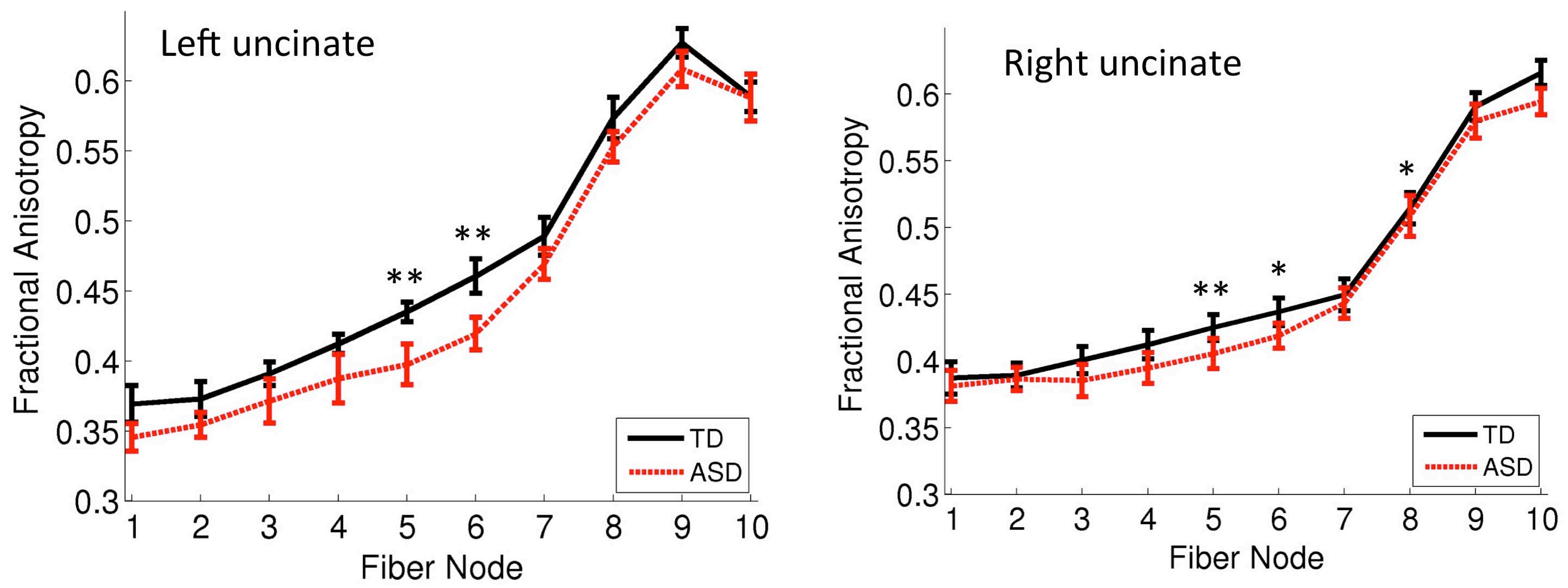